https://helda.helsinki.fi

Real numbers and projective spaces : Intuitionistic reasoning with undecidable basic relations

von Plato, Jan

2018-12

von Plato , J 2018 , ' Real numbers and projective spaces : Intuitionistic reasoning with

undecidable basic relations ' , Indagationes Mathematicae , vol. 29 , no. 6 , pp. 1546-1554 . https://doi.org/10.1016/j.

http://hdl.handle.net/10138/317010

https://doi.org/10.1016/j.indag.2017.10.012

cc_by_nc_nd

acceptedVersion

Downloaded from Helda, University of Helsinki institutional repository.

This is an electronic reprint of the original article.

This reprint may differ from the original in pagination and typographic detail.

Please cite the original version. 


\section{Accepted Manuscript}

Real numbers and projective spaces: Intuitionistic reasoning with undecidable basic relations

Jan von Plato

PII:

S0019-3577(18)30325-2

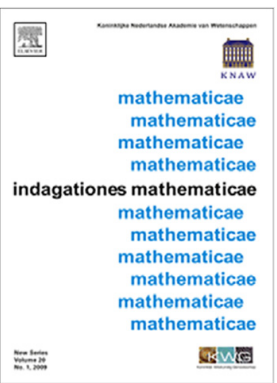

DOI: $\quad$ https://doi.org/10.1016/j.indag.2017.10.012

Reference: INDAG 591

To appear in: Indagationes Mathematicae

Please cite this article as: J. von Plato, Real numbers and projective spaces: Intuitionistic reasoning with undecidable basic relations, Indagationes Mathematicae (2018),

https://doi.org/10.1016/j.indag.2017.10.012

This is a PDF file of an unedited manuscript that has been accepted for publication. As a service to our customers we are providing this early version of the manuscript. The manuscript will undergo copyediting, typesetting, and review of the resulting proof before it is published in its final form. Please note that during the production process errors may be discovered which could affect the content, and all legal disclaimers that apply to the journal pertain. 


\title{
REAL NUMBERS AND PROJECTIVE SPACES: INTUITIONISTIC REASONING WITH UNDECIDABLE BASIC RELATIONS
}

\author{
Jan von Plato \\ University of Helsinki
}

\begin{abstract}
Brouwer introduced in 1924 the notion of an apartness relation for real numbers, with the idea that whenever it holds, a finite computation verifies it in contrast to equality. The idea was followed in Heyting's axiomatization of intuitionistic projective geometry. Brouwer in turn worked out an intuitionistic theory of "virtual order." It is shown that Brouwer's proof of the equivalence of virtual and maximal order goes only in one direction, and that Heyting's axiomatization needs to be made a bit stronger.
\end{abstract}

\section{Decidability: a common misconception about intuitionism}

In Emile Borel's version of constructivism from the first decade of the 20th century, there is a rather clear recognition that the equality of real numbers cannot be a decidable relation: For example, there is a way of computing what is known as Riemann's constant $C$, and the computation has so far given $0.4999 \ldots$. If every successive decimal is 9 , we have $C=0.5$, otherwise $\neg C=0.5$ holds. The condition for equality is expressed as a universal quantification over the decimals.

The same insight as in Borel got a more forceful expression in L. Brouwer's ideas about real numbers in the 1920's: He replaced equality of real numbers as a basic notion with the apartness of two reals, written $a \neq b$. That $a$ and $b$ are in this way distinct requires that there is a positive lower bound for their difference. Thus, a finite determination of values will verify apartness, though not falsify it, the precise contrary to the case of equality. The latter notion can now be defined as the negation of apartness. The point is how to reason with ideal objects and concepts such as real numbers and their properties and relations. If we follow the ideas of the logical positivists fashionable in the 1920s, there should be no undecidable basic relations: Their doctrine of verificationism requires a method for deciding truth, otherwise a notion is not meaningful. If such a method is assumed for the equality of reals, the law of excluded middle is justified: Then, its use gives $C=0.5 \vee \neg C=0.5$ with $C$ Riemann's constant and there are two cases, with two different consequences. As long as the value of $C$ remains undecided, nothing concrete follows from the cases. If instead the classical law is not allowed to enter, computability is maintained: logical reasoning will never lead from assumptions with a finitary meaning into something infinitistic. That is the whole point of constructive or intuitionistic logic. 
The standard view in the 1930s was that finitism and constructivism contain the requirement by which all basic relations be decidable and all functions computable. The former is an erroneous view to which one however could easily be led if one considered only intuitionistic arithmetic that can be formulated with a decidable equality as the only primitive relation, instead of also the intuitionistic theory of real numbers that cannot be based on a decidable equality, or the first intuitionistic axiomatization ever, Arend Heyting's 1925 system of intuitionistic projective geometry in which decidability of the basic apartness relations cannot be assumed. One of the first outside the intuitionist camp to realize the difference was Kurt Gödel whose lectures on intuitionism in Princeton in 1941 have been preserved in manuscript form. The extant text begins with two pages of improvements for the lectures, written in his Gabelsberger shorthand. One of the improvements is: "The belief is put aside that a system of axioms has an intuitionistic sense only if the basic concepts are decidable."

From the actual formal work of the intuitionists, Brouwer and his student Heyting in the first place, it can be seen that they required all functions to be computable, but not all basic relations to be decidable. So, why was there such a belief or requirement? One reason lies in the possibility to emulate operations with added basic relations. For example, one could substitute the operation of sum in arithmetic by a three-place relation written, say, $\Sigma(a, b, c)$ with the intended meaning that $c$ is the sum of $a$ and $b$.

\section{Apartness relations}

Brouwer's 1924 Intuitionistic division of the basic notions of mathematics contains a replacement of the equality relation of two real numbers by apart$n e s s, a \neq b$. The properties of apartness are, in logical notation:

1. $\neg a \neq a \quad$ irreflexivity

2. $a \neq b \supset a \neq c \vee b \neq c$ apartness axiom, co-transitivity

The second axiom is notable in a constructive context because it has a disjunction in a part of the formula (positive part) that cannot be rewritten in a constructively equivalent way without disjunction. It follows that whenever we have established $a \neq b$, any third real number $c$ can be taken and the two cases $a \neq c$ and $b \neq c$ formed.

By putting $a$ for $c$ in the second axiom, we get $a \neq b \supset a \neq a \vee b \neq a$, with the first disjunct negated in axiom 1 . Therefore symmetry, $a \neq b \supset$ $b \neq a$, follows.

Equality is a defined notion:

$$
a=b \equiv \neg a \neq b
$$

Reflexivity of equality is immediate from the definition, and symmetry and 
transitivity follow as contrapositions of symmetry of apartness and of axiom 2 , the latter in the "Euclidean" form $a=c \& b=c \supset a=b$, by Euclid's axiom in the Elements that says: "Two things equal to a third are equal among themselves."

The idea with an apartness relation is that one replaces an "infinitely precise" notion such as the equality of reals by a "finitely precise" apartness, in the sense that if $a \neq b$ happens to be the case, a finitely precise computation of the values of $a$ and $b$ verifies $a \neq b$, whereas the verification of $a=b$ requires infinite precision, whatever that may be. The "finite precision" idea validates the apartness axiom: given that $a$ and $b$ are apart, if $a \neq c$ cannot be decided, $c$ must be apart from $b$, and similarly for $b \neq c$.

In the 1924 paper, apartness was written as $a \# b$, most likely to make a difference to the standard definition as in $a \neq b \equiv \neg a=b$. Dana Scott suggested in 1968 to keep the standard notation, and for good reasons: If the equality relation happens to be decidable, the diaresis apartness and slashed apartness relations coincide. Following Scott, we have a uniform notation for the classical and constructive basic notions, and one just makes the choice as to which comes first in coneptual order.

\section{Virtual order}

Three years after the introduction of the apartness idea, Brouwer published another paper on elementary axiomatics, with the title "Virtual and inextensible order." The paper is typical of Brouwer in its idiosyncratic terminology and notation. Brouwer uses a notion of equality as distinct from identity. A set $P$, or "species" in Brouwer's terminology, with an order and equality relation is called partially projectibly ordered (teilweise geordnet projiziert), one with order and identity partially ordered, something like the difference within some of the more pedantic presentations of today between a preorder and an order. I shall use just an equality relation, as there is no role for a separate relation of identity, whatever that could be.

Brouwer begins with a basic axiom system, here written with a single equality relation:

$$
\begin{aligned}
& \text { A1. } r=r \\
& \text { A2. } r=s \& r=t \supset s=t \\
& \text { A3. } r>s \& r=t \& s=u \supset t>u \\
& \text { A4. } r>s \& s>t \supset r>t \\
& \text { A5. } \neg(r>s \& r=s)
\end{aligned}
$$

Brouwer has, of course no logical notation. He writes alternatively $r>s$ and $s<r$ and I follow him as it will make the proofs below handier to write down. Brouwer's first axiom gives that identity implies equality. Therefore, 
as identity is taken to be reflexive, his first axiom with equality in place of identity amounts to the reflexivity of equality. Brouwer's second axiom is the standard formulation of the transitivity of equality. As a consequence, symmetry of equality is not provable, something Brouwer fails to notice. I have repaired the matter by writing equality in the Euclidean style.

Brouwer's interest in the paper is to study two "additional axioms" of virtual order, ones he calls in German Ergänzungsaxiome and that can be given in terms of equality as:

$$
\begin{aligned}
& \text { A6. } \neg r>s \& \neg s>r \supset r=s \\
& \text { A7. } \neg r>s \& \neg r=s \supset s>r
\end{aligned}
$$

By the use of a weak order relation defined as in $r \leqslant s \equiv \neg r>s$, these can be written as:

$$
\begin{aligned}
& r \leqslant s \& s \leqslant r \supset r=s \\
& r \leqslant s \& \neg r=s \supset s>r
\end{aligned}
$$

Brouwer's main results are that axioms 6 and 7 follow from a single condition of maximality, called "inextensibility" of a virtual order $P$ in Brouwer (Unerweiterbarkeit). We assume that any such set is given by a domain of objects and a collection, possibly empty, of atomic relations:

Definition 1. Maximal order. A virtual order $P$ is maximal if, whenever a relation $r=s$ or $r>s$ can be consistently added to $P$, it is derivable by axioms A1-A5.

Brouwer's Satz 1 states that axiom 6 follows from axioms A1-A5 by the condition of maximality, and Satz 2 that axiom 7 follows. I shall present a detailed proof of the latter, just a reading of what Brouwer offers, even if he would hardly recognize the sameness of the proofs:

Proposition 2. Let a given virtual order $P$ be maximal. Then axiom 7 holds for $P$.

Proof. We prove axiom 7 in the form of $\neg s<r \& \neg r=s \supset r<s$.

Given $r$ and $s$, assume $\neg s<r \& \neg r=s$, and let the closure of the given relations $P$ with respect to axioms $\mathrm{A} 1-\mathrm{A} 5$ be denoted $\alpha$. It needs to be shown that $r<s$ can be consistently added to $\alpha$.

Let $\beta$ consist of relations $y<z$ such that: 1 . either $y<r$ or $y=r$ is in $\alpha$ and 2. either $s<z$ or $s=z$ is in $\alpha$.

Let $\gamma=\alpha+\beta$.

a) $\neg y=z$ and $\neg z<y$ are in $\alpha$. To prove this assume first $y=z$. Then we have four cases each of which turns out impossible:

1. $y<r, s<z: \quad y=z$ gives $z<r$, so $s<r$ against $\neg s<r$.

2. $y<r, s=z: \quad y=z$ gives $z<r$, so $s=z$ gives $s<r$. 
3. $y=r, s<z: \quad y=z$ gives $s<y$, then $y=r$ gives $s<r$.

4. $y=r, s=z: \quad y=z$ gives $z=y$, then $s=z$ gives $r=s$ against $\neg r=s$.

Next assume $z<y$, and there are again four cases that turn out impossible:

1. $y<r, s<z$ : Then $s<y$, so $s<r$.

2. $y<r, s=z$ : Then $s<y$, so $s<r$.

3. $y=r, s<z$ : Then $s<y$, so $s<r$.

4. $y=r, s=z$ : Then $s<y$, so $s<r$.

b) $\gamma$ is closed with respect to axioms A2-A4.

All equalities are in $\alpha$, so closure with respect to axiom A2 follows. For A3, we need some fresh variables, as in $a=b \& c=d \& a<c \supset b<d$. If $a<c$ is in $\alpha$ also $b<d$ is. If $a<c$ is in $\beta$, there are four cases as in a):

1. $a<r, s<c$ are in $\alpha$. Then $a=b$ gives $b<r$, and $c=d$ gives $s<d$, so $b<d$ is in $\gamma$.

Cases $2-4$ are similar.

If $r<s$ and $s<t$ are in $\gamma$ they are in $\alpha$ so that closure with respect to A4 follows.

It follows altogether that if $r<s$ is added to $\alpha$, the closure is included in $\gamma$.

c) For axiom 5, let $a=b$ be in $\alpha, a<b$ in $\beta$. The latter gives four cases:

1. $a<r, s<b$ are in $\alpha$. Then $b<r$ is in $\alpha$, so $s<r$, which is impossible.

Cases $2-4$ are similar.

Let $a<b$ be in $\beta, b<a$ in $\beta$. Then:

1. $a<r, s<b, b<s, r<a$ are in $\alpha$, which is impossible.

2. $a<r, s=b, b<s, r<a$ are in $\alpha$, which is impossible.

3. $a=r, s<b, b<s, r<a$ are in $\alpha$, which is impossible.

4. $a=r, s=b, b<s, r<a$ are in $\alpha$, which is impossible.

Cases 5-8 are symmetric with respect to $a, b$ and $b, a$.

It follows altogether that if $r<s$ is added to $\alpha$, the closure is included in $\gamma$.

We have shown that if $\neg s<r$ and $\neg r=s$, then $r<s$ is consistent, and therefore it is by maximality derivable.

QED.

The essential point is the inference from consistency (=not inconsistent) to derivability by maximality. The classical cases enter when $y \leqslant r$ and $s \leqslant z$ are defined by cases: $y<r$ or $y=r$, and $s<z$ or $s=z$. These are not decidable.

A proper intuitionistic formulation of axiom 7 should avoid the positive conclusion from negative conditions:

$$
\neg s<r \& \neg r=s \supset \neg \neg r<s
$$


An alternative writing without the double-negation is

$$
r \leqslant s \& \neg r=s \supset \neg s \leqslant r
$$

Both are easily provable, but Brouwer's axiom A7 with its positive conclusion from two negative conditions is too strong.

Brouwer's proof of his Satz 1 is almost identical to the one of Satz 2, up to the wording. This is strange, because Satz 1 is constructive, Satz 2 instead not. One wonders why Brouwer didn't base his treatment of order on the idea of apartness of two real numbers $a$ and $b$ : given that they are apart, there are two cases as in the refinement of apartness into two constructively decidable cases, the definition $a \neq b \equiv a<b \vee b<a$. Here the proper axiom would be $a<b \supset a<c \vee c<b$ from which the corresponding apartness axiom 2 in section 1 follows.

Brouwer completes his little article by a third theorem which claims that the converse to the theorems 1 and 2 holds: A virtual order is a maximal order. Brouwer's suggested proof aims to show the equivalence of maximally and axioms A6 and A7. It goes as follows (p. 408):

Let us assume that the relation $r=s$ (resp. the relation $r<s$ ) can be consistently added to the system $\alpha$ of the relations that obtain in the ordered projection $P$. Thence in system $\alpha$, both of the relations $r<s$ and $r>s$ (resp. the relations $r>s$ and $r=s$ ) are certainly contradictory [ungereimt]. Then as well, because the additional axiom 6 (resp. the additional axiom 7) holds for the relations in the ordered projection $P$, the relation $r=s$ (resp. the relation $r<s$ ) is contained in system $\alpha$. c. q. f. d.

This result is plain wrong, as the following counterexample shows:

Let the relations in $P$ be $a=a$ and $b=b$. Axiom 5 gives $\neg a<a$ and $\neg b<b$. This collection $\alpha$ is already closed with respect to axioms $1-7$, for the only axioms that can be applied are 1, 2, and 5, but with just two elements, nothing more follows. The rest of the axioms would need order relations. If now $a=b$ is added, consistency is maintained and $\neg a<b$ and $\neg b<a$ follow, but these were not in the original closure $\alpha$.

Brouwer states quite clearly that if $r=s$ can be consistently added, its contradictories by axiom $5 r<s$ and $r>s$ cannot. With the above example we would have, say, that the addition of $a<b$ to $a=a$ and $b=b$ leads to a contradiction. Brouwer's wording of the crucial erroneous step in the proof was: "Alsdann sind im System $\alpha$ sicher die beiden Relationen $r<s$ und $r>s$ ungereimt," not much of a step of proof to control for correctness, and Brouwer would have done better to resort to more formal methods in his proofs, namely those developed by his student Heyting. 


\section{Apartness in geometry}

Brouwer's idea of apartness was applied in the work of his student Arend Heyting, in the doctoral thesis Intuitionistische axiomatiek der projektieve meetkunde (Intuitionistic axiomatics of projective geometry) in 1925. I have studied it carefully in connection with my axiomatization of constructive geometry in 1995, but as most readers would fall short of controlling a discourse centered on that presentation, I refer instead to the shorter German article version, published in 1927 in the Mathematische Annalen with the title Zur intuitionistischen Axiomatik der projektiven Geometrie. As with Brouwer, Heyting's notation and terminology are idiosyncratic; the reason is in Brouwer's idea that mathematics has to be purified of old, bad connotations through new notation and a new terminology, one that in part consists of neologisms. Heyting uses the word species for the intuitionistic notion of a set. He gives an axiomatization for the projective space that consists of points, with lines, planes, and other geometric objects construed from these. I describe here the plane part that is structured as follows:

1. The basic objects are points that form the geometric space. Other geometric objects are given as species of points.

2. There are apartness and equality relations for points $A$ and $B$, written as $A \omega B$ and $A \sigma B$, respectively, and definitions of similar relations for species of points.

Lines are species of points. The geometric axioms are altogether 16, grouped from I to X; I shall describe the first ten axioms. The axioms for points are five, after a first one by which the space consists of points (p. 493):

Axiom I. The space is a mathematical species.

Axiom II. (Axiom of separation.)

IIa. $A \sigma B(A$ coincides with $B)$ and $A \omega B$ ( $A$ is apart from $B$ )

are invertible relations between the points $A$ and $B$.

IIb. $A \sigma A$ for every point $A$.

IIc. The relations $A \sigma B$ and $A \omega B$ are mutually exclusive.

IId. If $A \omega B$ is inconsistent, $A \sigma B$ holds.

IIe. If there is between the points $A$ and $B$ the relation $A \omega B$,

then for every point $C$ either $A \omega C$ or $B \omega C$.

The word for inconsistent in IId is "ungereimt," Brouwer's somewhat odd word construction. No logical notation is used, but it can be seen that by axioms IIc and IId, coincidence $A \sigma B$ is equivalent to the negation of apartness $A \omega B$, therefore a superfluous notion. Two theorems follow, the first the usual transitivity of equality, the second the substitution of equals in apartness, or from $A \omega B$ and $B \sigma C$ follows $A \omega C$. 
There follow now three axioms for lines, after one that produces two distinct points (ibid.):

Axiom III. Two points separate from each other can be determined.

Axiom IV. (Axiom of lines.) Lines are point species with the following properties:

IVa. If point $P$ belongs to line $l$, then every point that coincides with $P$ belongs to $l$.

IVb. Two points apart from each other determine a line that contains them both (i.e., one can determine a line $l$ that contains both of them, and every line that contains both of them is identical to $l$ ), their connecting line.

IVc. Every line contains at least three points apart from each other.

These axioms are followed by three lengthy definitions and axiom V (p. 494):

Definition. Point $P$ is apart from the point species $\alpha$ (also: lies outside the point species $\alpha$ ), if it is apart from each point of this species; we write in this case $P \omega \alpha$.

The point species $\alpha$ coincides with the point species $\beta$ or $\alpha \sigma \beta$ if every point of $\alpha$ coincides with some point of $\beta$ and every point of $\beta$ with some of $\alpha$.

The point species $\alpha$ is apart from the point species $\beta$ if $\alpha$ contains a point that is apart from $\beta$; we write then $\alpha \omega \beta$. This relation is not invertible.

Axiom V. A point can be determined outside every line.

The conceptual order of things in the axioms and definitions contains a crucial if somewhat subtle error that makes the axiomatization constructively too weak, as will be made clear in the next section.

Axiom IVb is clearly a construction postulate. An "infinitely precise" object is constructed that has some ideal, "infinitely precise" properties. Axioms III and V are clearly different from construction postulates, as they don't determine any unique new geometric objects from given ones. What to make of these axioms? Axiom IVc instead is formulated in the language of existence. What difference does that make to axioms III and V? Answers to these questions will require a little formal work: 


\section{Heyting's error in conceptual order}

A formal notation and some logic will be helpful to set things in order: We take points and lines and their apartness relations as primitives, with a new basic relation and notation:

$$
a \notin l \quad \text { point } a \text { is outside line } l
$$

Heyting had considered this notion in the 1950s but then discarded the idea as perhaps not worth the effort (see van Dalen). With outsideness as a primitive, incidence becomes a defined notion, in analogy to equality:

$$
a \in l \equiv \neg a \notin l
$$

Heyting's 1927 definition of apartness of a point from a species applied to $a$ and $l$ gives:

$$
\forall x(x \in l \supset a \neq x)
$$

A properly formulated constructive form of the principle of substitution of equals in the outsideness relation is:

$$
a \notin l \supset a \neq b \vee b \notin l
$$

The correctness of this axiom is seen similarly to Brouwer's apartness axiom. If $a \notin l$ and $b \in l$, the conclusion $a \neq b$ of Heyting's definitional axiom follows at once, but not the other way around, so we have:

Proposition 3. There is no way to arrive at the constructively correct disjunctive conclusion $a \neq b \vee b \notin l$ from $a \notin l$ by Heyting's notion of apartness of a point from a line.

What Heyting has is in substance $a \notin l \& b \in l \supset a \neq b$, analogous to the substitution axiom $a \notin l \& a=b \supset b \notin l$. This latter standard substitution principle of equals in the outsideness relation is constructively too weak.

Connecting lines of two distinct points were originally introduced by a postulate in Hilbert's Grundlagen der Geometrie of 1899, with the notation $A B$ and the condition of non-degeneracy $A \neq B$. This postulate was changed into a purely existential axiom in later editions, written in the present notation as:

$$
\forall x \forall y \exists z(x \neq y \supset x \in z \& y \in z)
$$

A formal notation for the line construction is $\ln (a, b)$, the connecting line of points $a$ and $b$. The connecting line has the ideal properties expressed by the axioms $a \neq b \supset a \in \ln (a, b), a \neq b \supset b \in \ln (a, b)$. A further property required is that the constructed object be unique:

$$
a \neq b \& a \in l \& a \in m \& b \in l \& b \in m \supset l=m
$$

Specifically, with $m \equiv \ln (a, b)$, we get

$$
a \neq b \& a \in l \& b \in l \supset l=\ln (a, b)
$$


Heyting's axioms III and V differ from axioms for constructions; they are in some sense purely existential, not replaceable by constructions. They can be formulated as:

III: $\exists x \exists y . x \neq y$ and $\mathrm{V}: \forall x \exists y . y \notin x$

In axiom $\mathrm{V}$, the sorts of objects can be read off from the way the relation is written, with a point as the left member and a line as right in the outsideness relation.

The standard intuitionistic explanation of the quantifiers gives:

If $\forall x \exists y A(x, y)$, there is a function $f$ such that $A(x, f(x))$

This kind of explanation fails for the axiom $\forall x \exists y . y \notin x$. Heyting's axioms III and $\mathrm{V}$ are instead rather like the things required in the proof of Euclid's first proposition: "On a given line segment to produce an equilateral triangle." The proof starts with: "Let $A B$ be the given line segment." This is clearly an existential assumption, and $A$ and $B$ act as eigenvariables in an inference from the asumption. As a result, the construction applies to any line $A B$.

Heyting's axioms III and V express tasks that can be met by "finitary capacities," as in:

Two distinct points $a, b$ can be determined.

To mark three points a,b,c such that they form a triangle.

The points $a, b, c$ are "generic," i.e., the only thing we know about them is $a \neq b$ and $c \notin \ln (a, b))$. Existential axioms such as Heyting's $\exists x \exists y . x \neq y$ and $\forall x \exists y . y \notin x$ have precisely this effect when the existential quantifiers are instantiated by eigenvariables. Heyting's geometry gives at the same time an example of an intuitionistic theory in which the existence property fails; a formal proof of this failure can be given for the axiom system of the next Section.

\section{The axioms of intuitionistic projective geometry}

We finish this note by a summary of the proper axioms of intuitionistic projective geometry:

Basic relations $a \neq b, l \neq m, a \notin l$

Apartness axioms for point and line apartness:

$$
\begin{aligned}
& \neg a \neq a, \neg l \neq l \\
& a \neq b \supset a \neq c \vee b \neq c, \quad l \neq m \supset l \neq n \vee m \neq n
\end{aligned}
$$

Connecting line and intersection point constructions:

$\ln (a, b), p t(l, m)$, conditions $a \neq b, l \neq m$

Incidence properties: 


$$
a \in \ln (a, b), b \in \ln (a, b), p t(l, m) \in l, p t(l, m) \in m
$$

Substitution of equals:

$$
a \notin l \supset a \neq b \vee b \notin l, \quad a \notin l \supset l \neq m \vee a \notin m
$$

Uniqueness:

$$
a \neq b \& l \neq m \supset a \notin l \vee a \notin m \vee b \notin l \vee b \notin m
$$

The classical contrapositive is Skolem's axiom, as in 2.4.(C):

$$
a \in l \& a \in m \& b \in l \& b \in m \supset a=b \vee l=m
$$

The classical versions of substitutions are:

$$
a \in l \& a=b \supset b \in l, \quad a \in l \& l=m \supset a \in m
$$

Non-collinearity:

$$
\exists x \exists y \exists z(x \neq y \& z \notin \ln (x, y))
$$

The last axiom is purely existential; still, the existence property obviously fails. Assume it held, and there would be a derivation of $a \neq b \& c \notin \ln (a, b)$ for some three points $a, b, c$. Then even $a \neq b$ would be derivable. By the general result of my second paper, such a derivation would have the subterm property: all terms in the derivation are visible in the open assumptions, here none, or the conclusion. It is seen at once that there can be no derivation with this property for the formula $a \neq b$, and we have:

Proposition 4. Intuitionistic plane projective geometry does not have the existence property.

\section{References:}

Brouwer, L. (1924) Intuitionistische Zerlegung mathematischer Grundbegriffe. As reprinted in Brouwer (1975), pp. 275-280.

Brouwer, L. (1927) Virtuelle Ordnung und unerweiterbare Ordnung. As reprinted in Brouwer (1975), pp. 406-408.

Brouwer, L. (1975) Collected Works, vol. 1, North-Holland, Amsterdam.

van Dalen, D. (1996) 'Outsideness' as a primitive notion in constructive projective geometry. Geometriae Dedicata, vol. 60, pp. 107-111.

van Dalen, D. (2013) L.E.J. Brouwer: Topologist, Intuitionist, Philosopher. Springer.

Heyting, A. (1925) Intuitionistische axiomatiek der projektieve meetkunde. P. Noordhoff, Groningen.

Heyting, A. (1927) Zur intuitionistischen Axiomatik der projektiven Geometrie. Mathematische Annalen, vol. 98, pp. 491-538. 
Hilbert, D. (1899) Grundlagen der Geometrie. Teubner, Leipzig. Several later editions.

von Plato, J. (1995) The axioms of constructive geometry. Annals of Pure and Applied Logic, vol. 76, pp. 169-200.

von Plato, J. (2010) Combinatorial analysis of proofs in projective and affine geometry. Annals of Pure and Applied Logic, vol. 162, pp. 144-161.

Scott, D. (1968) Extending the topological interpretation to intuitionistic analysis. Compositio Mathematica, vol. 20, pp. 194-210. 